\title{
The Sharia Compliance of Gold-Backed- Cryptocurrency: Analysis of Volatility and Risk
}

\author{
Fauziyah Adzimatinur ${ }^{1}$, Vigory Gloriman Manalu², Faishal Rahimi $^{3}$ \\ ${ }^{123}$ Universitas Kuningan, Indonesia \\ \{f.adzimatinur@uniku.ac.id\}
}

\begin{abstract}
Cryptocurrency in the perspective of Islam has currently become a subject undergoing intense study among researchers. The Islamic perspective of money, has specific characteristics and requirements, such as stability and is based on assets. These characteristics also applied to the Cryptocurrency as it becomes the latest trend of payment. There are several Cryptocurrencies that has gold back up. This research aims to evaluate the volatility and risk possessed by the gold-backed Cryptocurrencies compared to other cryptocurrencies (Bitcoin and Ethereum), S\&P500, and gold. The stability of Gold-Backed-Cryptocurrency is analyzed using ARCH-GARCH method. The data used in this research is daily data of Gold-Backed-Cryptocurrency price for a year. The results will show us how close the gold-backed-cryptocurrencies to the Sharia Compliance. The results show that gold-backed-cryptocurrencies are more volatile than Bitcoin, Ethereum, S\&P500. This shows how unstable they are and greater risk they possess. The results conclude that gold-backed-cryptocurrencies are nowhere close to the sharia compliance. Other characteristics have to be fulfilled such as authorities and free of speculation.
\end{abstract}

Keywords: Cryptocurrency; gold-backed; Sharia Compliance

\section{Introduction}

Cryptocurrency has been rapidly growing in this era. This virtual currency is based on cryptographic evidence that allows two parties to transact directly with each other without the need for a trusted third party (for example a bank) [1]. Cryptocurrency is defined as a digital currency in which encryption techniques are used to regulate the formation of currency units and verify the transfer of funds, cryptocurrency operates independently of the central bank. Cryptocurrency is a digital asset designed as a medium of exchange using cryptography to secure transactions and is difficult to fake because of its security features. Cryptocurrency is not issued by a central authority, making it theoretically immune to government interference [2].

Islam regulates the lives of its people in all aspects, including muamalah, in this case the problem of transactions using money. Islam views money only as a medium of exchange, so the demand for money is only to fulfill transaction needs. In Islamic theory, there is no idea of money demand for speculation, because speculation is not permitted and Islam makes property the object of zakat. Money belongs to society and must always revolve in the economy, the faster the money rotates in the economy, the greater the wealth and welfare of society. There is no established time value of money in the Islamic definition, but rather the economic value of time, i.e. the value of money may not be dependent on increasing time, since money has no time value, but rather time has an economic value. 
There are several studies that have been carried out regarding the use of digital transactions or non-cash transactions from an Islamic perspective. One of the things that is of concern is the use of credit cards for transactions. Quoted from Monzer Kahf, one of the Islamic economic leaders, stated that signing a credit card contract and using it so as to result in payments that generate interest is haram because it is included in usury. Whereas signing a contract then using it and paying within the grace period and not making cash withdrawals is allowed because it is a contract that gives the option of dealing or not dealing with interest.

Muamar (2017) [3] conducted a study on electronic money (e-money) in the perspective of maqashid sharia. From the research results, it is generally found that electronic money is in accordance with the maqashid sharia. This conformity is obtained by the fulfillment of the principles of maintaining property and benefit. However, electronic money which is unregistered considered incompatible with the maqashid sharia because it does not have a PIN, so it still causes harm if the card is stolen or lost.

As with other digital transactions, it is necessary to study cryptocurrency from an Islamic perspective. There are differences in transaction procedures using traditional currencies and cryptocurrencies. In traditional transactions, transactions between two parties are carried out through a trusted system provided by the banking sector. This transaction uses a centralized management system created by a banking institution. The security of this transaction is monitored by a banking institution. The central bank has the authority to control the amount of money in circulation with monetary policy.

There are two main views. One side argues that cryptocurrency is a bubble without real assets that is sure to end up bursting. The other side argues that the cryptocurrency market will be an avenue that will give millions of people the opportunity to participate in a global financial network worth tens of trillions of dollars.

In the transaction procedure using cryptocurrency, if one party wants to transfer digital currency to another party, the transaction must pass through the block chain. Block chain is in a ledger system that is monitored and validated by users involved in the ledger validation system using a computer system. Cryptocurrency makes it easy to transfer funds between two parties in one transaction. Cryptocurrencies have no physical form, only balances that are stored in a public ledger in a cloud facility. Transactions of Cryptocurrency can only be carried out via electronic means, usually allowing for instant transactions and unlimited transfer of ownership.

Technological developments cannot be avoided, especially in this digital era. The community needs an easy and practical means of transactions and investment. Therefore, there is a need for a sharia-compliant cryptocurrency as an alternative to cryptocurrency for sharia loyalists. To build cryptocurrency a sharia-based, things to pay attention to, of course, are related to sharia-compliant aspects, especially maqashid sharia on cryptocurrency.

Bakar (2017) [4] examines the framework, cryptocurrency especially Bitcoin in the perspective of Islamic finance, his research provides an overview of several things in cryptocurrency (in this case Bitcoin) that are incompatible with Islamic principles, including the aspect of gharar which is widely contained in cryptocurrency. Bitcoin's value is not tied to real assets or government regulations and laws, and there is a risk of being hacked and there is no clarity about the real account holder.

Siswantoro (2020) [5] evaluated the suitability of cryptocurrency as money from the Islamic perspective. Money, in the Islamic perspective, has specific characteristics and requirements, such as stability and is based on assets. Cryptocurrency may not fulfil this as it has queries as money from the Islamic perspective. The research method applied data of 23 cryptocurrency prices and related information. The result shows that cryptocurrency is hugely 
volatile and has limits to being called 'money,' as it is limited and used for speculation, which is prohibited in Islam

From the previous researches, one of the criteria of money in Islam is it should be based on real asset. Some cryptocurrencies have real asset as their base, such as gold. Two of the top gold-backed cryptocurrencies are DigiXGlobal (DGX) and Gold Coin (GLC) [6]. This study aims to evaluate the stability of gold-backed cryptocurrencies compared with Bitcoin and Ethereum as two of the biggest cryptocurrencies and S\&P500. The novelty of this study is determining whether the asset based cryptocurrency can be another alternative for shariacompliant cryptocurrency as it fulfills one of the criteria's of money in Islam. Precious metals like gold have always been key commodities in the trade market. With the soaring gold prices, the cryptocurrency trade market is turning to gold-backed cryptocurrency tokens. While this idea is relatively new, it has prospects of making cryptocurrency more stable [6].

\section{Method}

The volatility of the return of cryptocurrency, gold-backed cryptocurrency, and S\&P500 will be analyzed using ARCH/GARCH method using Eviews 8. Return is a description of the rate of return obtained by companies, individuals and institutions from the results of their investment choices [7]. Return is used as a benchmark for consideration before making decisions in investing activities. Return is calculated from the difference in the natural logarithm of today's and previous stock closing price movements [8]. Return is formulated as follows:

Note:

$$
R_{t}=\operatorname{Ln}\left(X_{t} / X_{t-1)}\right.
$$

$R t$ : return in day- $\mathrm{t}$

$X t$ : closing price in day- $t$

$X t-1$ : closing price in day-t-1

The return volatility of the variables is investigated using the ARCH-GARCH model. The ARCH-GARCH model was introduced by Engle in 1982. ARCH stands for autoregressive conditional heteroscedasticity. This model was developed primarily to address the problem of volatility in economic and business data, particularly in the financial sector. This volatility is reflected in the error variance that does not meet the homoscedasticity assumption (variance of constant error over time). Bollerslev in 1986 then developed this model known as GARCH, which stands for generalized autoregressive conditional heteroscedasticity [8].

The ARCH (m) model assumes that the variation fluctuation data is influenced by the fluctuation of the data amounting to " $\mathrm{m}$ " in the previous period. GARCH (r, m) assumes that the fluctuation of data variation is influenced by the fluctuation of a number of " $m$ " data in the previous period and the volatility of a number of $r$ data. The following is the general form of the GARCH (r, m) model in this study:

$$
h_{t}=K+\delta_{1} h_{t-1}+\delta_{2} h_{t-2}+\cdots+\delta_{r} h_{t-r}+\alpha_{1} \varepsilon_{t-1}^{2}+\alpha_{2} \varepsilon_{t-2}^{2}+\cdots+\alpha_{m} \varepsilon_{t-m}^{2}
$$

In the general form above, there are two equations in the GARCH (r, m) model, one for the conditional mean and one for the conditional variance. The ARCH coefficient shows the volatility in the previous period and the GARCH coefficient shows the persistence of shocks through volatility. Meanwhile, $\mathrm{h}_{-} \mathrm{t}$ is the return for the variables studied at time $\mathrm{t}$; $\mathrm{K}$ is a constant; $\alpha_{-} 1, \alpha_{-} 2, \ldots, \alpha_{-} \mathrm{m}$ is the estimated $\mathrm{m}$ order coefficient $\delta_{-} 1, \delta_{-} 2, \ldots, \delta_{-} \mathrm{r}$ is the 
estimated coefficient of $r$ order. The data used in this study is daily data of each variable in 1 year period (November 2019 - November 2020).

Table 1. Data Source

\begin{tabular}{l|l}
\hline \multicolumn{1}{c}{ Variable } & \multicolumn{1}{c}{ Source } \\
\hline S\&P 500 & Yahoo Finance \\
Bitcoin (Btc) & Yahoo Finance \\
Ethereum (Eth) & Yahoo Finance \\
Digixglobal (Dgx) & Coingecko.Com \\
Gold coin (Glc) & Coingecko.Com \\
\hline
\end{tabular}

The research framework of this study is as follows:

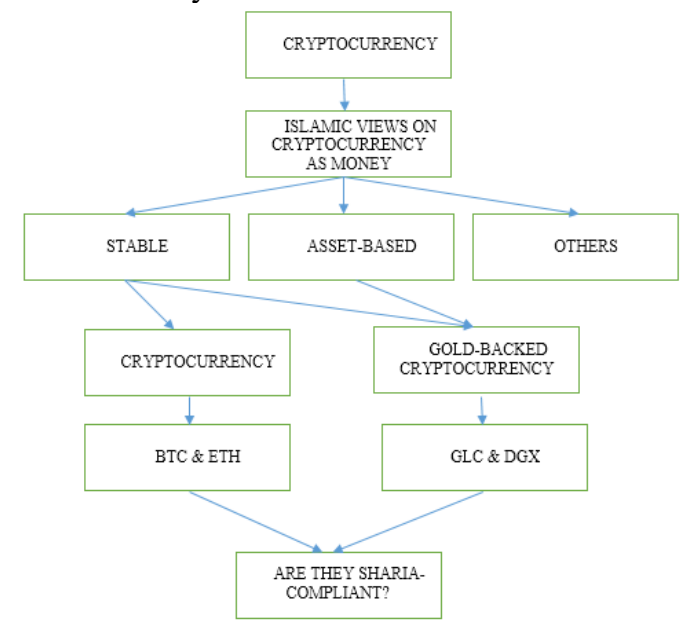

Fig 1. Research Framework

\section{Result and Discussion}

The return volatility in the studied variable can be analyzed using the ARCH/GARCH model by using the best model. The best model is chosen based on the smallest value of Akaike Information Criterion (AIC) and Schwarz Criterion (SC). The best models in this study are as follows:

Table 2. ARCH/GARCH Model

\begin{tabular}{llrrr}
\hline \multirow{2}{*}{ Variable } & \multirow{2}{*}{ Model } & \multicolumn{3}{c}{ Coefficient } \\
\cline { 3 - 5 } & & \multicolumn{1}{c}{$\mathrm{K}$} & \multicolumn{1}{c}{$\delta_{1}$} & $\alpha_{1}$ \\
\hline S\&P 500 & GARCH $(1,1)$ & 0.00000692 & 0.366952 & 0.680857 \\
\hline GLC & ARCH 1 & 0.048894 & 0.193824 \\
\hline DGX & GARCH $(1,1)$ & 0.00000231 & 0.019774 & 1.020571 \\
\hline BTC & GARCH $(1,1)$ & 0.000041 & 0.391804 & 0.749564 \\
\hline ETH & GARCH $(1,1)$ & 0.0000721 & 0.161229 & 0.849201 \\
\hline
\end{tabular}

\subsection{The Return Volatility of Each Variables}

Figure $1-5$ shows the Eviews 8 output for the return volatility of each variable. Figure 1 shows the return volatility of S\&P 500. The figure shows that the return is volatile in 75 th period and gradually becoming more stable. The 75th period is the period where the 
coronavirus outbreak takes place. In that time, almost every aspect in economy became unstable as there are uncertainties in the economy as a result of the pandemic.
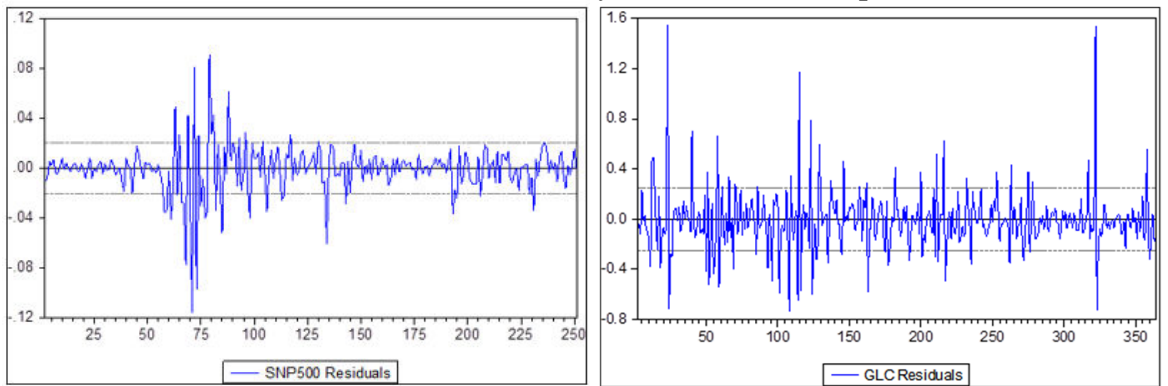

Fig 1. Return Volatile of S\&P 500 and GLC

Figure 2 shows the return volatility of Gold Coin (GLC). The figure shows that the return is quite volatile in a whole year studied. The highest volatility happened in the beginning and in the ending of the period. In the middle of the period the return is more stable than that in the beginning and ending. The return of this cryptocurrency is too volatile, therefore it possesses high risk. This means that GLC does not fulfill the criteria of money in Islam, so that it cannot be an alternative for the sharia-compliant cryptocurrency. Figure 3 shows the return volatility of another gold-backed cryptocurrency studied in this research. The figure shows that the return volatility of DGX is more stable than that in GLC. It shows that although it is considered volatile at the beginning of the period, but the volatility is not as high as in GLC. However, in the near-ending of the period, there happens high volatility of the return. This shows that investing in DGX possesses high risk. From the figure, we can conclude that although one of money criteria's in Islam is asset based [4], DGX and GLC cannot be alternative for the sharia-compliant cryptocurrency.
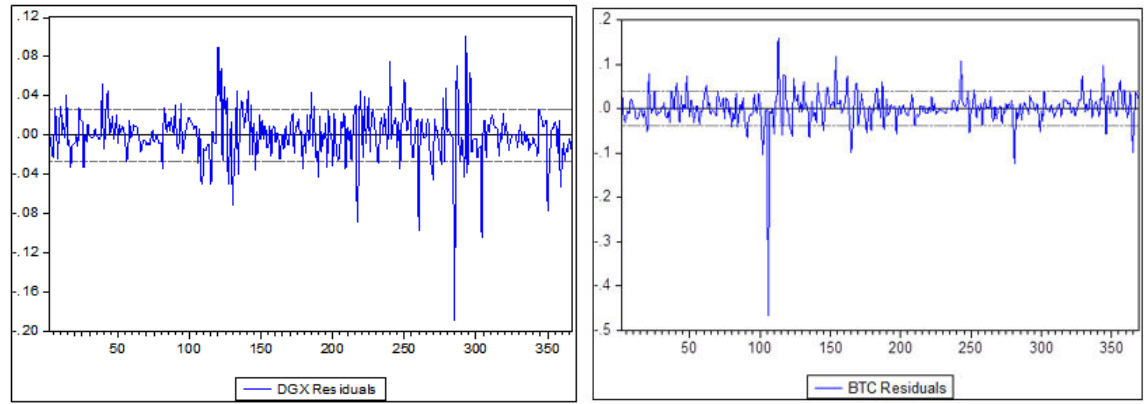

Fig 2. Return Volatile of DGX and BTC

Figure 4 shows the return volatility of the biggest cryptocurrency i.e Bitcoin. It shows that in the beginning of the period, there happens the highest volatility. The high volatility might be affected by the economy uncertainty because of the coronavirus outbreak. However, in the following period, the volatility started to stabilize. When comparing the return volatility of Bitcoin and S\&P500, the result is in line with the research conducted by Siswantoro (2020) [5]. The research stated that the Cryptocurrency cannot be categorized as money in Islam as it possesses high volatility and high risk. The return volatility of Ethereum is shown in Figure 5. The movement of the volatility along the period studied is similar with the Bitcoin. This might happen because these two giant cryptocurrencies were affected by the pandemic at the beginning as the economy uncertainty occurred. However, in the following period the 
volatility became more stable. The return volatility of these two cryptocurrencies (Bitcoin and Ethereum) is more stable than that possessed by the gold-backed cryptocurrencies.

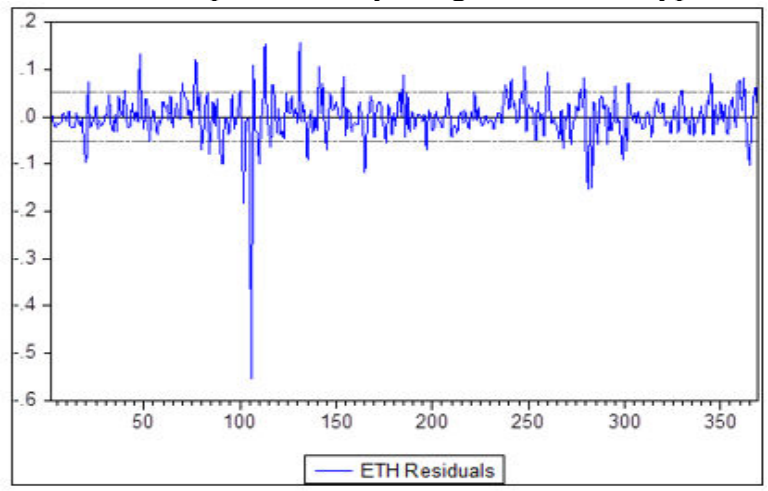

Fig 3. Return Volatility of ETH

\section{Conclusion}

Money, in the Islamic perspective, has specific characteristics and requirements, such as stability and is based on assets [5]. According to the characteristics, this study aims to analyze the stability of the gold-backed cryptocurrency. However, the results show that neither goldbacked cryptocurrencies studied (GLC and DGX) fulfill the stability characteristic. This means the cryptocurrencies are nowhere near the sharia-compliant cryptocurrencies. Same thing goes to Bitcoin and Ethereum, although they are less volatile than the gold-backed cryptocurrencies, the volatility is high. Therefore, they are unstable and possess high risk. As for S\&P 500, the index is more stable compared to the cryptocurrencies studies. This concludes that for now, cryptocurrencies are nowhere near sharia-compliant. A lot of aspects still need to be fulfilled beside stability and real asset based. Those aspects such as free of gharar and speculation, there needs to be authority to regulate the money, and other additional aspects included charity [9].

\section{References}

[1] L. Kristoufek, "BitCoin meets Google Trends and Wikipedia: Quantifying the relationship between phenomena of the Internet era," Sci. Rep., vol. 3, pp. 1-7, 2013, doi: 10.1038/srep03415.

[2] R. Böhme, N. Christin, B. Edelman, and T. Moore, "Bitcoin: Economics, Technology, and Governance," vol. 29, no. 2, pp. 213-238, 2015.

[3] Afif Muamar dan Ari Salman Alparisi, "Electronic money (E-money) dalam perspektif maqashid syariah,” J. Islam. Econ. Lariba, vol. 3, no. 2, pp. 76-77, 2017.

[4] N. Abu Bakar, S. Rosbi, and K. Uzaki, "Cryptocurrency Framework Diagnostics from Islamic Finance Perspective: A New Insight of Bitcoin System Transaction,” Int. J. Manag. Sci. Bus. Adm., vol. 4, no. 1, pp. 19-28, 2017, doi: 10.18775/ijmsba.1849-5664-5419.2014.41.1003.

[5] D. Siswantoro, R. Handika, and A. F. Mita, "The requirements of cryptocurrency for money, an Islamic view," Heliyon, vol. 6, no. 1, p. e03235, 2020, doi: 10.1016/j.heliyon.2020.e03235. 
[6] "Top Five Gold-Backed Cryptocurrency in 2020," 2020. [Online]. Available: https://www.fintechnews.org/top-five-gold-backed-cryptocurrency-in-2020/. [Accessed: 30-Nov2020].

[7] I. Fahmi, Pengantar Pasar Modal. Bandung: Alfabeta, 2012.

[8] M. Firdaus, Ekonometrika: Suatu Pendekatan Aplikatif, 2nd ed. Jakarta: Bumi Aksara, 2011.

[9] M. M. Billah, Halal Cryptocurrency Management. Palgrave Macmillan, 2019. 